\title{
Complex Lagrange multiplier approach to the design of arbitrary complex coefficient FIR digital filters
}

\author{
Jong-Jy Shyu \\ Department of Computer Science and Engineering, Tatung Institute of Technology. Taipei, Taiwan, Republic of China \\ Soo-Chang Pei \\ Department of Electrical Engineering, National Taiwan University, Taipei, Taiwan, Republic of China \\ Kuo-Chang $\mathrm{Fu}$ \\ Department of Computer Science and Engineering, Tatung Institute of Technology, Taipei, Taiwan, Republic of China
}

Received 3 December 1992

Revised 5 April 1993

\begin{abstract}
The Lagrange multiplier approach has been widely used to design maximally flat, real coefficients FIR digital filters and differentiators. This paper extends this approach to design arbitrary complex coefficient FIR digital filters with arbitrary frequency response or derivative constraints. The method can easily be extended to design arbitrary two-dimensional complex FIR digital filters with arbitrary frequency response or derivative constraints, too. Several examples are presented to demonstrate the effectiveness of the approach.

Zusammenfassung. Die Lagrangesche Multiplikatorenmethode wird oft für den Entwurf maximal flacher FIR-Digitalfilter und Differentiatoren mit reellen Koeffizienten verwendet. In der vorliegenden Arbeit wird die Lagrangesche Multiplikatorenmethode auf den Entwurf beliebiger komplexer FIR-Digitalfilter mit beliebigem Frequenzgang bzw. beliebigen Ableitungs-Nebenbedingungen erweitert. Die Methode kann auch in einfacher Weise auf den Entwurf beliebiger zweidimensionaler komplexer FIR-Digitalfilter mit beliebigem Frequenzgang bzw. beliebigen Ableitungs-Nebenbedingungen erweitert werden. Mehrere Beispiele zeigen die Effektivität der Methode.

Résumé. L’approche du multiplieur de Lagrange a été largement utilisée pour définir le plat maximum, les coefficients réels des filtres et des différentiateurs digitaux FIR. Dans cet article, l'approche du multiplieur de Lagrange est étendue pour définir les coefficients complexes des filtres digitaux FIR possédant une fréquence de réponse arbitraire ou des contraintes dérivées. La méthode peut être facilement étendue pour définir des filtres digitaux FIR complexes à deux dimensions avec une fréquence de réponse arbitraire ou des contraintes dérivées. Plusieurs exemples sont présentés permettant de démontrer l'efficacité de cette approche
\end{abstract}

Keywords. Lagrange multiplier approach; complex FIR digital filter; one-sided differentiator; two-dimensional FIR filter.

\section{Introduction}

Recently, the design of arbitrary complex coefficient FIR digital filters has been widely considered $[1,7,8]$. In [7], the real oriented eigenfilter method is used to design complex FIR digital filters, while the complex eigenfilter approach [8] and the complex weighted least-squares approach [1] design the complex coefficient

Correspondence to: Dr. Jong-Jy Shyu, Department of Computer Science and Engineering, Tatung Institute of Technology, 40 Chungshan N. Rd., 3rd Sec., Taipei, Taiwan, 104, Republic of China. 
FIR filters directly. However, it is difficult to impose the frequency response or derivative constraints for the above methods.

In this paper, we will propose a complex Lagrange multiplier approach for the design of arbitrary complex coefficient FIR digital filters with additional frequency response or derivative constraints. The Langrange multiplier approach was originally used to design maximally flat, real coefficient FIR filters and differentiators $\left[\begin{array}{ll}3 & 6\end{array}\right]$. The method is based on minimizing the integrated square error for the real magnitude response over the passband and stopband while imposing derivative constraints. In this paper, the complex Lagrange multiplier approach, extended from the real case, is used to design complex coefficient FIR digital filters whose frequency responses approximate the arbitrary desired complex frequency responses while imposing arbitrary frequency or derivative constraints. The method can also be extended easily to design twodimensional FIR filters with arbitrary frequency response or derivative constraints. Section 2 formulates the design problem and the extension to the two-dimensional case is shown in Section 3, in which several examples are presented to demonstrate the effectiveness of the approach. Finally, the conclusions are given in Section 4.

\section{The complex Lagrange multiplier approach}

For a complex coefficient FIR digital filter, its frequency response can be characterized by

$H(\omega)=\sum_{n=0}^{N-1} a(n) \mathrm{e}^{-\mathrm{j} n \omega}$,

where $N$ is the filter length and the filter coefficients $a(n)$ 's are complex-valued. Defining the column vectors

$\boldsymbol{A}=\left[\begin{array}{lllll}a(0) & a(1) & a(2) & \ldots & a(N-1)\end{array}\right]^{\mathrm{T}}$

and

$C(\omega)=\left[\begin{array}{lllll}1 & \mathrm{e}^{\mathrm{j} \omega} & \mathrm{e}^{\mathrm{j} 2 \omega} & \ldots & \mathrm{e}^{\mathrm{j}(N-1) \omega}\end{array}\right]^{\mathrm{T}}$,

(1) can be rewritten as

$H(\omega)=\boldsymbol{A}^{\mathrm{T}} \boldsymbol{C}^{*}(\omega)=\boldsymbol{C}^{\mathrm{H}}(\omega) \boldsymbol{A}$,

where $\mathrm{T}$ and $\mathrm{H}$ denote the transpose and the Hermitian transpose operators, respectively, and $*$ denotes the complex conjugate operator. Now we wish to use (4) to approximate the desired arbitrary complex frequency response $D(\omega)$.

Using these notations, the integrated square complex error over the $k$ th passband with band-edges $\omega_{p_{1}}$ and $\omega_{p_{2}}$ is

$$
\begin{aligned}
e_{p_{k}} & =\int_{\omega_{p_{1}}}^{\omega_{p_{2}}}\|D(\omega)-H(\omega)\|^{2} \mathrm{~d} \omega \\
& =s_{k}+\boldsymbol{P}_{k}^{\mathrm{H}} \boldsymbol{A}+\boldsymbol{A}^{\mathrm{H}} \boldsymbol{P}_{k}+\boldsymbol{A}^{\mathrm{H}} \boldsymbol{Q}_{\boldsymbol{p}_{k}} \boldsymbol{A},
\end{aligned}
$$

where

$s_{k}=\int_{\omega_{p_{i}}}^{\omega_{p_{2}}} D^{*}(\omega) D(\omega) \mathrm{d} \omega$,

$\boldsymbol{P}_{k}=\int_{\omega_{p_{i}}}^{\omega_{p_{2}}}-D(\omega) \boldsymbol{C}(\omega) \mathrm{d} \omega$

Signal Processing 
and

$Q_{p_{k}}=\int_{\omega_{p_{1}}}^{\omega_{p_{2}}} \boldsymbol{C}(\omega) \boldsymbol{C}^{\mathbf{H}}(\omega) \mathrm{d} \omega$.

Similarly, the integrated square complex error over the $l$ th stopband with band-edges $\omega_{s_{1}}$ and $\omega_{s_{2}}$ is

$e_{s_{l}}=\int_{\omega_{s_{l}}}^{\omega_{s_{2}}}\|H(\omega)\|^{2} \mathrm{~d} \omega=A^{\mathrm{H}} Q_{s_{l}} A$,

where

$\boldsymbol{Q}_{s_{l}}=\int_{\omega_{s_{1}}}^{\omega_{s_{2}}} \boldsymbol{C}(\omega) C^{\mathrm{H}}(\omega) \mathrm{d} \omega$

Assume there are $K$ passbands and $L$ stopbands in the desired filter; then the total error to be minimized is

$$
\begin{aligned}
e & =\alpha_{1} e_{p_{1}}+\alpha_{2} e_{p_{2}}+\cdots+\alpha_{K} e_{p_{K}}+\beta_{1} e_{s_{1}}+\beta_{2} e_{s_{2}}+\cdots+\beta_{L} e_{s_{L}} \\
& =s+\boldsymbol{P}^{\mathrm{H}} \boldsymbol{A}+\boldsymbol{A}^{\mathrm{H}} \boldsymbol{P}+\boldsymbol{A}^{\mathrm{H}} \boldsymbol{Q} \boldsymbol{A},
\end{aligned}
$$

where

$$
\begin{aligned}
& s=\alpha_{1} s_{1}+\alpha_{2} s_{2}+\cdots+\alpha_{K} s_{K}, \\
& P=\alpha_{1} \boldsymbol{P}_{1}+\alpha_{2} \boldsymbol{P}_{2}+\cdots+\alpha_{K} \boldsymbol{P}_{K}
\end{aligned}
$$

and

$\boldsymbol{Q}=\alpha_{1} \boldsymbol{Q}_{p_{1}}+\alpha_{2} \boldsymbol{Q}_{p_{2}}+\cdots+\alpha_{K} \boldsymbol{Q}_{p_{\mathrm{K}}}+\beta_{1} \boldsymbol{Q}_{s_{1}}+\beta_{2} \boldsymbol{Q}_{s_{2}}+\cdots+\beta_{L} \boldsymbol{Q}_{s_{L}}$,

in which $\alpha_{i}, i=1,2, \ldots, K$, and $\beta_{i}, i=1,2, \ldots, L$, are the weighting constants.

The desired filter passband performance is attained by imposing frequency response or derivative constraints $F$ at a discrete set of points $\omega_{0}, \omega_{1}, \ldots, \omega_{J}$ in the passband, i.e.

$F_{m j}\left[H\left(\omega_{j}\right)\right]=g_{m j}, \quad 0 \leqslant m \leqslant M$ and $0 \leqslant j \leqslant J$,

where $M$ denotes the order of constraints at a particular frequency $\omega_{j}$. Equation (15) can be rewritten as

$B^{\mathrm{H}} \boldsymbol{A}=\boldsymbol{G}$,

where

$\boldsymbol{B}=\left[\begin{array}{llllllllll}F_{00}[C(\omega)] & \ldots & F_{M 0}[C(\omega)] & F_{01}[C(\omega)] & \ldots & F_{M 1}[C(\omega)] & \ldots & F_{0 J}[C(\omega)] & \ldots & F_{M J}[C(\omega)]\end{array}\right]$

and

$\boldsymbol{G}=\left[\begin{array}{llllllllll}g_{00} & \cdots & g_{M 0} & g_{01} & \cdots & g_{M 1} & \cdots & g_{0 J} & \cdots & g_{M J}\end{array}\right]^{\mathrm{T}}$.

Hence, the design of filters with frequency response or derivative constraints can be formulated as a quadratic programming problem:

Minimize $e=s+\boldsymbol{P}^{\mathrm{H}} \boldsymbol{A}+\boldsymbol{A}^{\mathrm{H}} \boldsymbol{P}+\boldsymbol{A}^{\mathrm{H}} \boldsymbol{Q} \boldsymbol{A}$

Subject to $\boldsymbol{B}^{\mathrm{H}} \boldsymbol{A}=\boldsymbol{G}$. 
Let the complex Lagrange multiplier vector be

$\lambda=\left[\begin{array}{llllllllll}\lambda_{00} & \ldots & \lambda_{M 0} & \lambda_{01} & \ldots & \lambda_{M 1} & \ldots & \lambda_{0 J} & \ldots & \lambda_{M J}\end{array}\right]^{\mathrm{T}}$

then the Lagrangian function is

$$
\begin{aligned}
\Lambda(\boldsymbol{A}, \lambda) & =s+\boldsymbol{P}^{\mathrm{H}} \boldsymbol{A}+\boldsymbol{A}^{\mathrm{H}} \boldsymbol{P}+\boldsymbol{A}^{\mathrm{H}} \boldsymbol{Q} \boldsymbol{A}-\lambda^{\mathrm{H}}\left(\boldsymbol{B}^{\mathrm{H}} \boldsymbol{A}-\boldsymbol{G}\right) \\
& =s+\boldsymbol{P}^{\mathrm{H}} \boldsymbol{A}+\boldsymbol{A}^{\mathrm{H}} \boldsymbol{P}+\boldsymbol{A}^{\mathrm{H}} \boldsymbol{Q} \boldsymbol{A}-\left(\boldsymbol{A}^{\mathrm{H}} \boldsymbol{B}-\boldsymbol{G}^{\mathrm{H}}\right) \lambda
\end{aligned}
$$

because $\lambda^{\mathrm{H}}\left(\boldsymbol{B}^{\mathrm{H}} \boldsymbol{A}-\boldsymbol{G}\right)$ is a real-valued function. The necessary and sufficient conditions for optimality are

$\nabla_{A} \Lambda=0$

and

$\nabla_{\lambda} \Lambda=0$,

where $\nabla$ denotes a gradient operator, which leads to

$\left[\begin{array}{cc}-\boldsymbol{Q} & \boldsymbol{B} \\ \boldsymbol{B}^{\mathrm{H}} & \mathbf{0}\end{array}\right]\left[\begin{array}{l}\boldsymbol{A} \\ \lambda\end{array}\right]=\left[\begin{array}{l}\boldsymbol{P} \\ \boldsymbol{G}\end{array}\right]$

where $\mathbf{0}$ is a zero matrix. Solving (24), we can obtain the closed-form solutions

$\boldsymbol{A}=\boldsymbol{Q}^{-1} \boldsymbol{B}\left(\boldsymbol{B}^{\mathrm{H}} \boldsymbol{Q}^{-1} \boldsymbol{B}\right)^{-1} \boldsymbol{G}+\boldsymbol{Q}^{-1}\left[\boldsymbol{B}\left(\boldsymbol{B}^{\mathrm{H}} \boldsymbol{Q}^{-1} \boldsymbol{B}\right)^{-1} \boldsymbol{B}^{\mathrm{H}} \boldsymbol{Q}^{-1}-\boldsymbol{I}\right] \boldsymbol{P}$

and

$\lambda=\left(B^{\mathrm{H}} Q^{-1} \boldsymbol{B}\right)^{-1}\left(G+B^{\mathrm{H}} Q^{-1} P\right)$,

where $\boldsymbol{I}$ is an $N \times N$ identity matrix. Notice that $\boldsymbol{Q}$ is a Toeplitz Hermitian symmetric matrix and $\boldsymbol{B}^{\mathrm{H}} \boldsymbol{Q}^{-1} \boldsymbol{B}$ is a Hermitian symmetric matrix; the Levinson recursion [2] can be used to compute $\boldsymbol{Q}^{-1}$ and $\left(\boldsymbol{B}^{\mathrm{H}} \boldsymbol{Q}^{-1} \boldsymbol{B}\right)^{-1}$ very effectively such that the solutions of $A$ and $\lambda$ do not need the inverse computations.

EXAMPLE 1. Design of a single-passband complex filter.

This example deals with the design of a single-passband complex filter with passband $\left[\omega_{p_{1}}, \omega_{p_{2}}\right]$, stopband $\left[\omega_{s_{1}}, \omega_{s_{2}}\right]$, and a constant group delay $\tau$ in the passband, i.e.

$D(\omega)= \begin{cases}\mathrm{e}^{-\mathrm{j} \tau \omega}, & \omega_{p_{1}} \leqslant \omega \leqslant \omega_{p_{2}} \\ 0, & \omega_{s_{1}} \leqslant \omega \leqslant \omega_{s_{2}}\end{cases}$

The closed forms for all elements of $\boldsymbol{P}$ and $\boldsymbol{Q}$ can easily be derived and are given, respectively, by

$p_{i}= \begin{cases}\alpha_{1}\left(\omega_{p_{1}}-\omega_{p_{2}}\right) & \text { if } i=\tau, \\ \alpha_{1}\left[\frac{\sin \left((i-\tau) \omega_{p_{1}}\right)-\sin \left((i-\tau) \omega_{p_{2}}\right)}{i-\tau}+\mathrm{j} \frac{\cos \left((i-\tau) \omega_{p_{2}}\right)-\cos \left((i-\tau) \omega_{p_{1}}\right)}{i-\tau}\right] & \text { if } i \neq \tau,\end{cases}$

Signal Processing 
where $0 \leqslant i \leqslant N-1$, and

$$
q_{i_{1} i_{2}}=\left\{\begin{array}{l}
\alpha_{1}\left(\omega_{p_{2}}-\omega_{p_{1}}\right)+\beta_{1}\left(\omega_{s_{2}}-\omega_{s_{1}}\right) \quad \text { if } i_{1}=i_{2}, \\
\alpha_{1}\left[\frac{\sin \left(\left(i_{1}-i_{2}\right) \omega_{p_{2}}\right)-\sin \left(\left(i_{1}-i_{2}\right) \omega_{p_{1}}\right)}{i_{1}-i_{2}}+\mathrm{j} \frac{\cos \left(\left(i_{1}-i_{2}\right) \omega_{p_{1}}\right)-\cos \left(\left(i_{1}-i_{2}\right) \omega_{p_{2}}\right)}{i_{1}-i_{2}}\right] \\
+\beta_{1}\left[\frac{\sin \left(\left(i_{1}-i_{2}\right) \omega_{s_{2}}\right)-\sin \left(\left(i_{1}-i_{2}\right) \omega_{s_{1}}\right)}{i_{1}-i_{2}}+\mathrm{j} \frac{\cos \left(\left(i_{1}-i_{2}\right) \omega_{s_{1}}\right)-\cos \left(\left(i_{1}-i_{2}\right) \omega_{s_{2}}\right)}{i_{1}-i_{2}}\right]
\end{array}\right.
$$

where $0 \leqslant i_{1}, i_{2} \leqslant N-1$. For example, when $N=31, \tau=12, \omega_{p_{1}}=-0.1 \pi, \omega_{p_{2}}=0.3 \pi, \omega_{s_{1}}=0.4 \pi$, $\omega_{s_{2}}=1.8 \pi, \alpha_{1}=\beta_{1}=1$, and we hope to impose third-order derivative constraints on the designed frequency response at $\omega_{0}=0.1 \pi$, i.e.

$\boldsymbol{B}=\left.\left[\begin{array}{llll}\boldsymbol{C}(\omega) & \frac{\mathrm{d}}{\mathrm{d} \omega} \boldsymbol{C}(\omega) & \frac{\mathrm{d}^{2}}{\mathrm{~d} \omega^{2}} \boldsymbol{C}(\omega) & \frac{\mathrm{d}^{3}}{\mathrm{~d} \omega^{3}} \boldsymbol{C}(\omega)\end{array}\right]\right|_{\omega=\omega_{0}}$

and

$\boldsymbol{G}=\left[\begin{array}{lll}\mathrm{e}^{-\mathrm{j} \tau \omega} & \frac{\mathrm{d}}{\mathrm{d} \omega} \mathrm{e}^{-\mathrm{j} \tau \omega} \frac{\mathrm{d}^{2}}{\mathrm{~d} \omega^{2}} \mathrm{e}^{-\mathrm{j} \tau \omega} \frac{\mathrm{d}^{3}}{\mathrm{~d} \omega^{3}} \mathrm{e}^{-\mathrm{j} \tau \omega}\end{array}\right]_{\omega=\omega_{0}}^{\mathrm{T}}$

The resultant magnitude response and group-delay response are shown in Figs. 1 (a) and 1(b), respectively, while the traces of complex errors in the passband and stopband are shown in Figs. 1(c) and 1(d), respectively. For checking the maximal property, the passband detail of the absolute complex error is shown in Fig. 1(e). The filter coefficients are given in Table 1.

EXAMPLE 2. Design of a positive-sided differentiator.

The desired response of a positive-sided differentiator is given by

$D(\omega)= \begin{cases}\mathrm{j} \omega \mathrm{e}^{-\mathrm{j} \tau \omega}, & 0 \leqslant \omega \leqslant \omega_{p}, \\ 0, & \omega_{s} \leqslant \omega \leqslant 2 \pi,\end{cases}$

where $\tau$ is the desired group delay in the passband. The closed forms of the elements of $\boldsymbol{P}$ are given by

$$
p_{i}=\left\{\begin{array}{l}
\mathrm{j} x_{1} \frac{\omega_{p_{1}}^{2}-\omega_{p_{2}}^{2}}{2} \text { if } i_{1}=\tau, \\
\alpha_{1}\left\{\left[\frac{\sin \left((i-\tau) \omega_{p_{2}}\right)-\sin \left((i-\tau) \omega_{p_{1}}\right)}{(i-\tau)^{2}}+\frac{\omega_{p_{1}} \cos \left((i-\tau) \omega_{p_{1}}\right)-\omega_{p_{2}} \cos \left((i-\tau) \omega_{p_{2}}\right)}{i-\tau}\right]\right. \\
\left.\quad+\mathrm{j}\left[\frac{\cos \left((i-\tau) \omega_{p_{1}}\right)-\cos \left((i-\tau) \omega_{p_{2}}\right)}{(i-\tau)^{2}}+\frac{\omega_{p_{1}} \sin \left((i-\tau) \omega_{p_{1}}\right)-\omega_{p_{2}} \sin \left((i-\tau) \omega_{p_{2}}\right)}{i-\tau}\right]\right\}
\end{array}\right.
$$




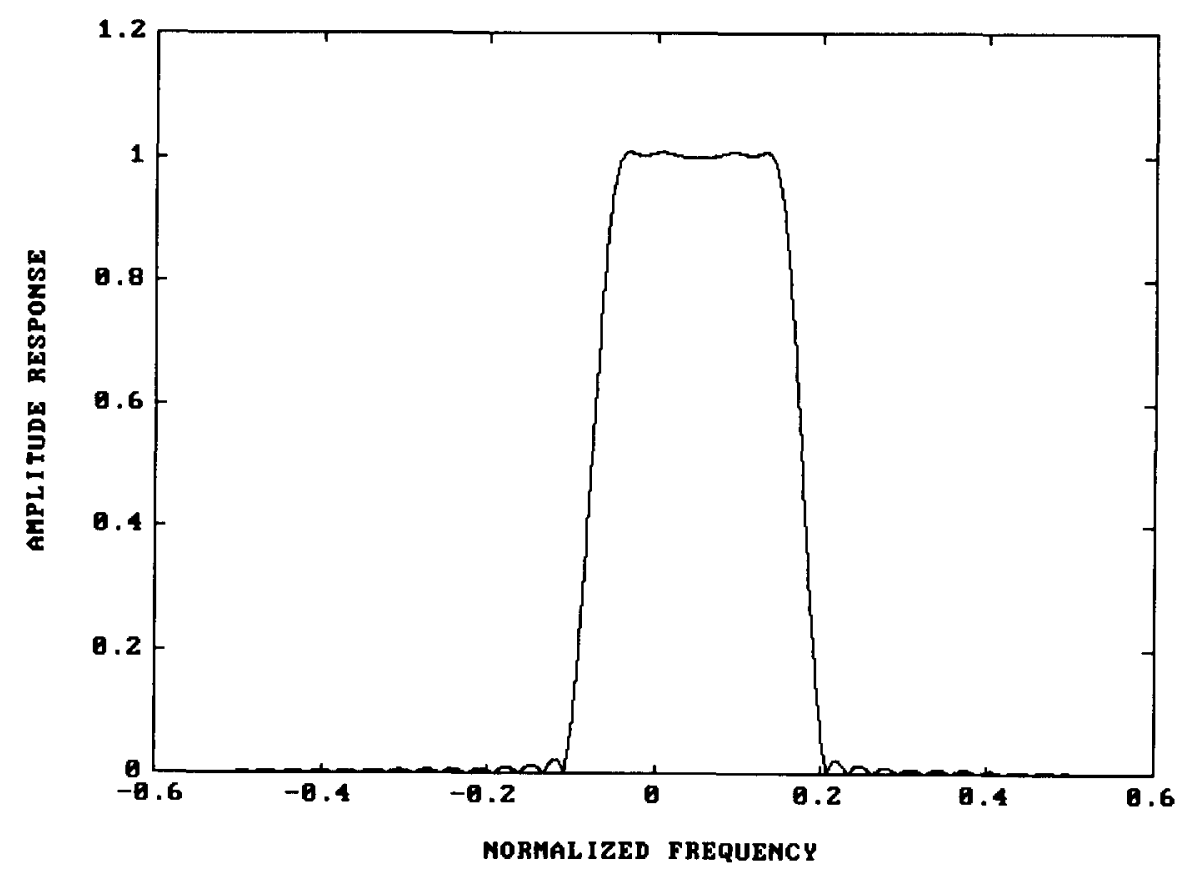

Fig. 1. Example 1: design of a single-passband filter. (a) Magnitude response.

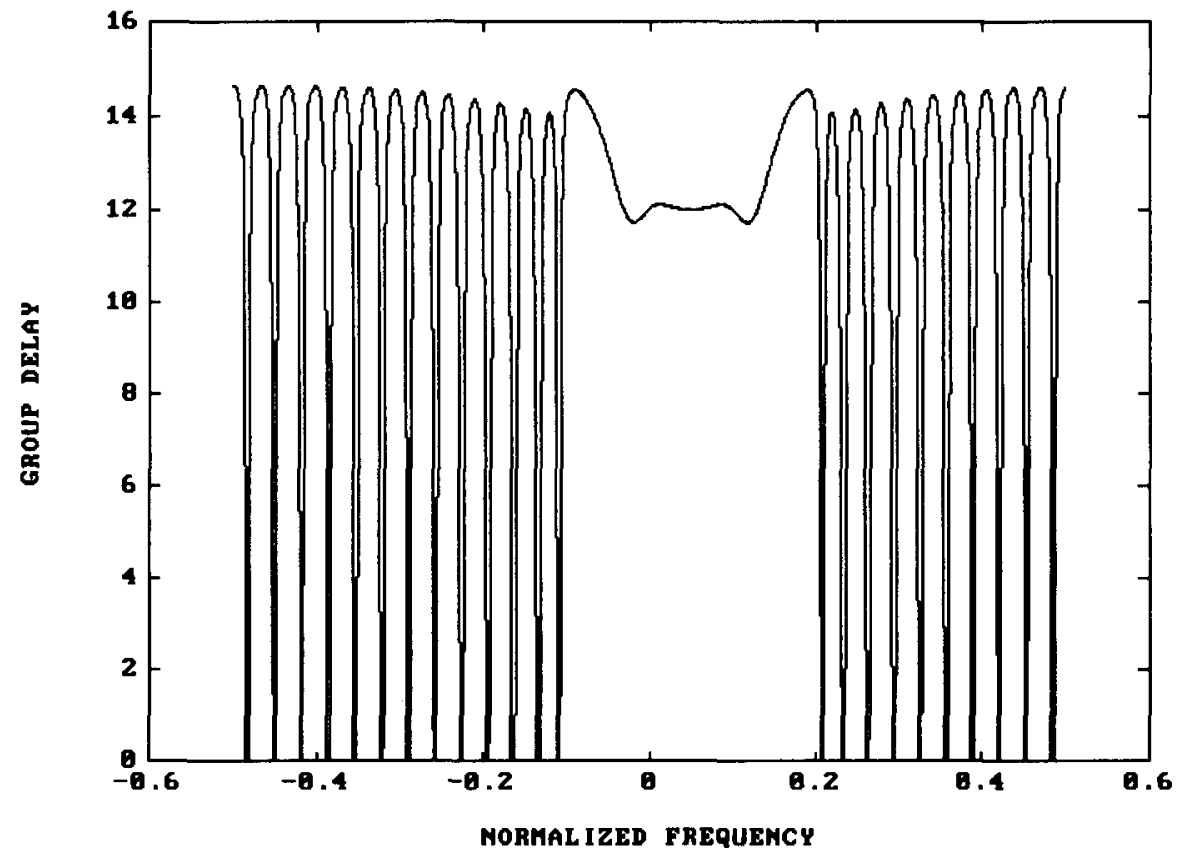

Fig. 1. Example 1. (b) Delay response. 


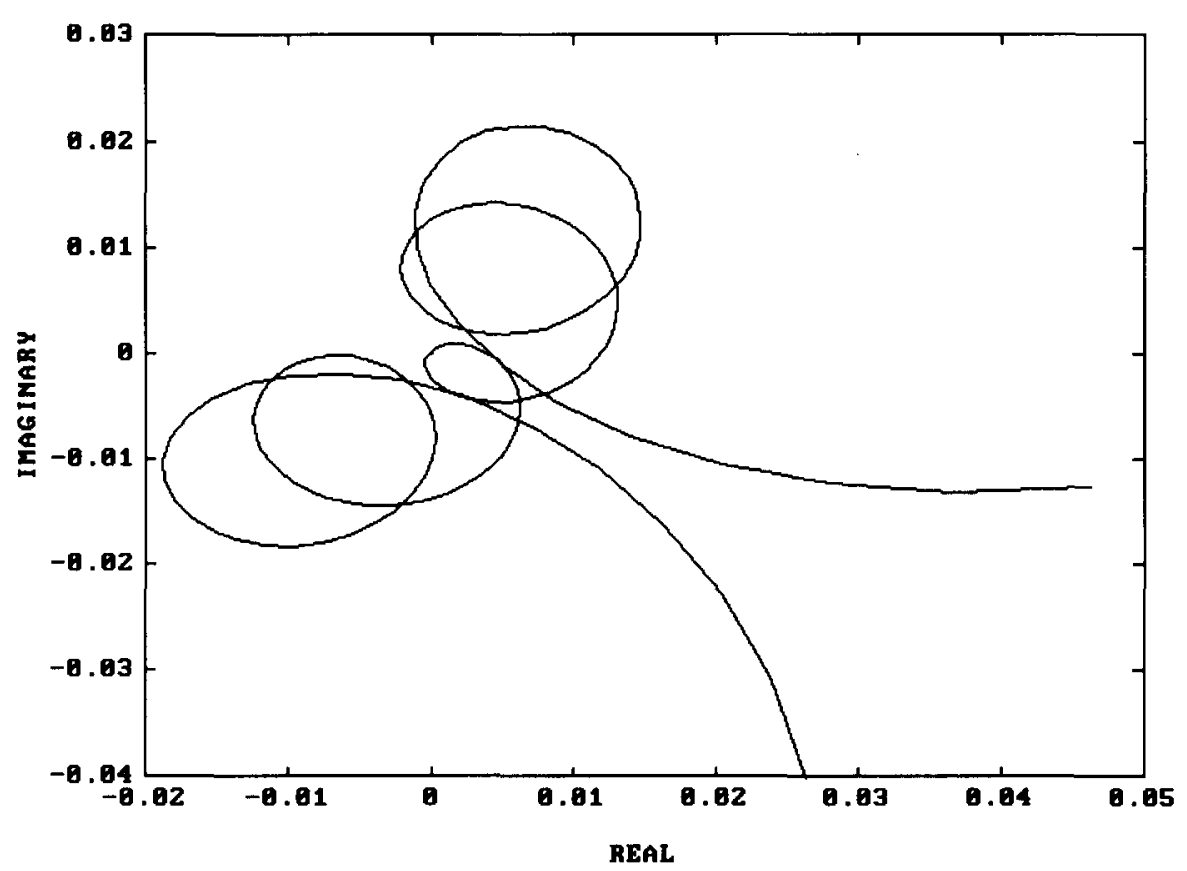

Fig. 1. Example 1. (c) Trace of passband complex error.

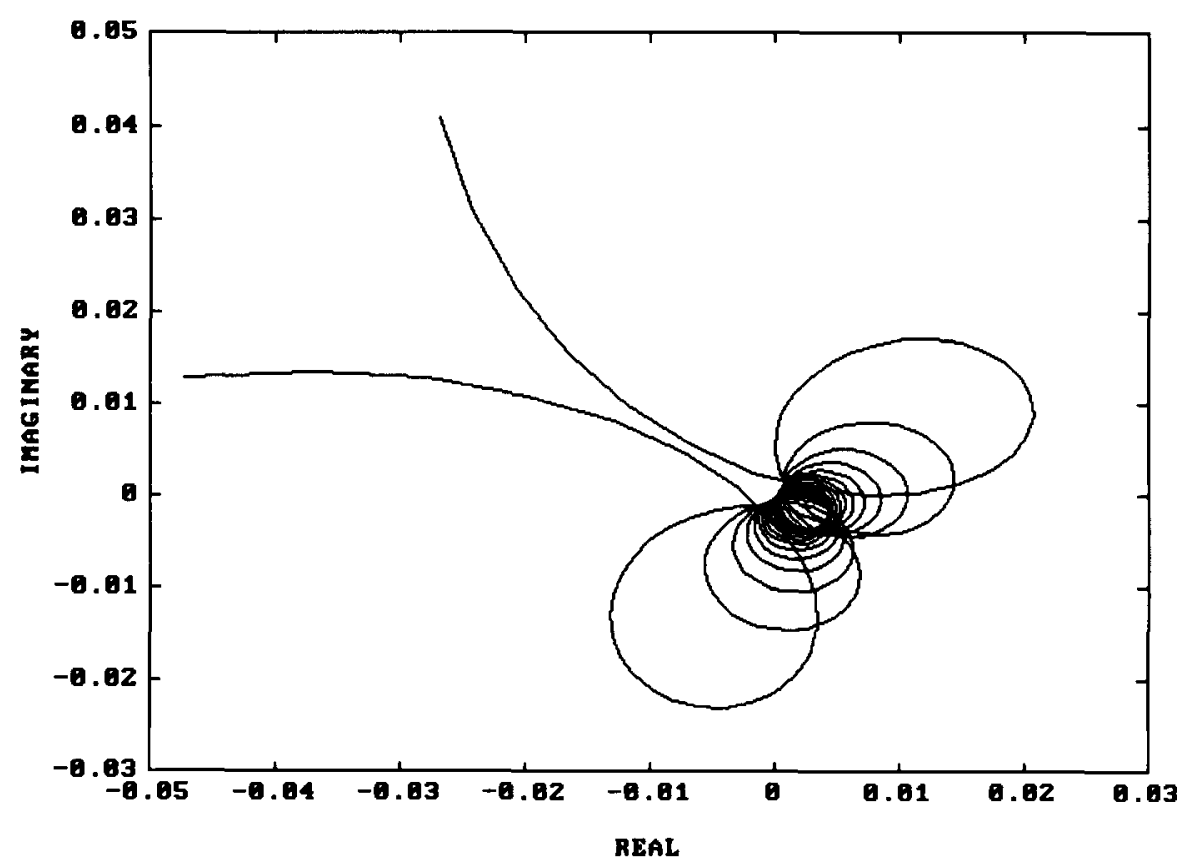

Fig. 1. Example 1. (d) Trace of stopband complex error. 


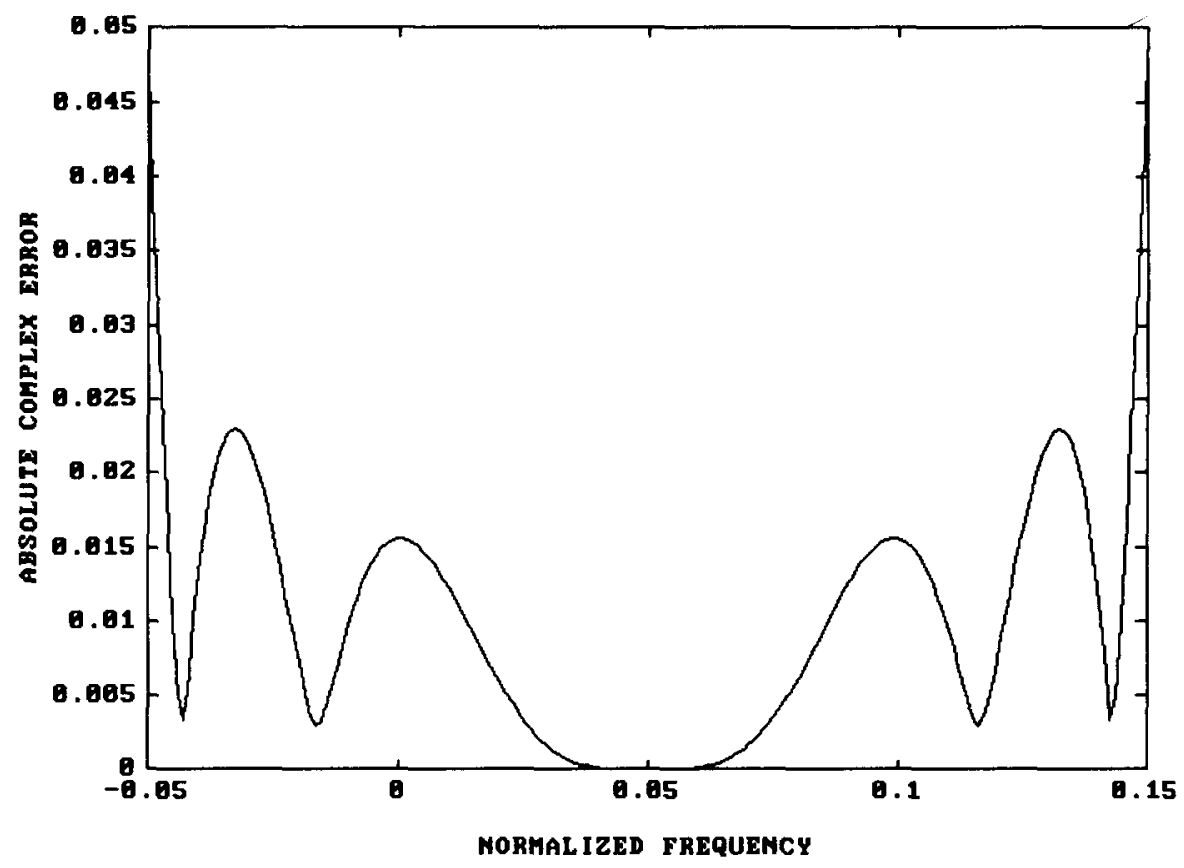

Fig. 1. Example 1. (e) Absolute passband complex error.

where $0 \leqslant i \leqslant N-1$, and the elements of $Q$ can be obtained from (29). For example, when $N=31, \tau=12$, $\omega_{p}=0.8 \pi, \omega_{s}=0.9 \pi, \alpha_{1}=\beta_{1}=1$, and third-order derivative constraints at $\omega_{0}=0.4 \pi$ are imposed, i.e.

$\boldsymbol{G}=\left.\left[\begin{array}{lll}\mathrm{j} \omega \mathrm{e}^{-\mathrm{j} \tau \omega} & \frac{\mathrm{d}}{\mathrm{d} \omega} \mathrm{j} \omega \mathrm{e}^{-\mathrm{j} \tau \omega} \frac{\mathrm{d}^{2}}{\mathrm{~d} \omega^{2}} \mathrm{j} \omega \mathrm{e}^{-\mathrm{j} \tau \omega} \frac{\mathrm{d}^{3}}{\mathrm{~d} \omega^{3}} \mathrm{j} \omega \mathrm{e}^{-\mathrm{j} \tau \omega}\end{array}\right]^{\mathrm{T}}\right|_{\omega=\omega_{0}}$,

and the matrix $\boldsymbol{B}$ is the same as (30). The resultant magnitude and group-delay responses are shown in Figs. 2(a) and 2(b), respectively, while the traces of complex errors in the passband and stopband are given in Figs. 2(c) and 2(d), respectively. Meanwhile, the absolute complex error in the passband is shown in Fig. 2(e), and the filter coefficients are tabulated in Table 1.

\section{Design of two-dimensional complex FIR digital filters}

The design method described in Section 2 for one-dimensional complex filters can be extended to two-dimensional complex filter design. For a two-dimensional complex FIR digital filter, its frequency response can be characterized as

$H\left(\omega_{1}, \omega_{2}\right)=\sum_{n_{1}=0}^{N_{1}-1} \sum_{n_{2}=0}^{N_{2}-1} a\left(n_{1}, n_{2}\right) \mathrm{e}^{-\mathrm{j} n_{1} \omega_{1}} \mathrm{e}^{-\mathrm{j} n_{2} \omega_{2}}$

For simplicity, let $N_{1}=N_{2}=N$. Defining

$\boldsymbol{A}=\left[\begin{array}{llll}\boldsymbol{A}_{0}^{\mathrm{T}} & \boldsymbol{A}_{1}^{\mathrm{T}} & \cdots & \boldsymbol{A}_{N-1}^{\mathrm{T}}\end{array}\right]^{\mathrm{T}}$

Signal Processing 
Table 1

The filter coefficients in Examples 1 and 2

\begin{tabular}{|c|c|c|}
\hline$n$ & Single-passband filter & Positive-sided differentiator \\
\hline 0 & $2.1874603 E-03+j-1.5892829 E-03$ & $1.9125712 E-03+j-8.3262432 E-04$ \\
\hline 1 & $-3.3287901 \mathrm{E}-03+\mathrm{j} \quad 1.0815895 \mathrm{E}-03$ & $-3.2459907 \mathrm{E}-03+\mathrm{j}-1.2403623 \mathrm{E}-03$ \\
\hline 2 & $-9.5635426 \mathrm{E}-03+\mathrm{j} \quad 1.7283538 \mathrm{E}-16$ & $5.7898939 \mathrm{E}-03+\mathrm{j}-2.7699634 \mathrm{E}-03$ \\
\hline 3 & $-9.1932304 \mathrm{E}-03+\mathrm{j}-2.9870616 \mathrm{E}-03$ & $-8.8080210 E-03+j \quad 2.6862519 E-03$ \\
\hline 4 & $3.3264989 \mathrm{E}-04+\mathrm{j} \quad 2.4168429 \mathrm{E}-04$ & $1.0946473 E-02+j-1.3428802 E-02$ \\
\hline 5 & $1.0924354 \mathrm{E}-02+\mathrm{j}$ & $-1.0850652 E-02+j \quad 1.7300140 E-02$ \\
\hline 6 & $1.0656202 E-02+j$ & $6.0766987 \mathrm{E}-03+\mathrm{j}-3.8098856 \mathrm{E}-02$ \\
\hline 7 & $1.2491364 \mathrm{E}-16+\mathrm{j}$ & $6.6719832 \mathrm{E}-03+\mathrm{j} \quad 4.3235514 \mathrm{E}-02$ \\
\hline 8 & $-1.2469557 \mathrm{E}-04+\mathrm{j}$ & $-3.2481560 \mathrm{E}-02+\mathrm{j}-7.6643882 \mathrm{E}-02$ \\
\hline 9 & $3.7291495 \mathrm{E}-02+\mathrm{j}-5.1327340 \mathrm{E}-02$ & $8.1095897 E-02+j \quad 6.3066780 E-02$ \\
\hline 10 & $1.1682468 \mathrm{E}-01+\mathrm{j}-8.4878100 \mathrm{E}-02$ & $-1.7786522 \mathrm{E}-01+\mathrm{j}-1.1905434 \mathrm{E}-01$ \\
\hline 11 & $2.0548855 E-01+j-6.6767278 E-02$ & $4.4738204 \mathrm{E}-01+\mathrm{j}-1.8184355 \mathrm{E}-01$ \\
\hline 12 & $2.5105222 \mathrm{E}-01+\mathrm{j} \quad 4.0708403 \mathrm{E}-17$ & $2.7950285 \mathrm{E}-02+\mathrm{j} \quad 6.3682202 \mathrm{E}-01$ \\
\hline 13 & $2.2254288 \mathrm{E}-01+\mathrm{j} \quad 7.2308565 \mathrm{E}-02$ & $-5.0011747 \mathrm{E}-01+\mathrm{j}-1.6470585 \mathrm{E}-01$ \\
\hline 14 & $1.3719956 \mathrm{E}-01+\mathrm{j} \quad 9.9681316 \mathrm{E}-02$ & $2.2166466 \mathrm{E}-01+\mathrm{j}-1.5082530 \mathrm{E}-01$ \\
\hline 15 & $4.7686140 \mathrm{E}-02+\mathrm{j}$ & $-1.1182902 \mathrm{E}-01+\mathrm{j} \quad 1.0504266 \mathrm{E}-01$ \\
\hline 16 & $-1.2633942 E-05+j-3.8883276 E-05$ & $4.8026128 \mathrm{E}-02+\mathrm{j}-1.2351230 \mathrm{E}-01$ \\
\hline 17 & $2.6098102 \mathrm{E}-16+\mathrm{j}-4.8752984 \mathrm{E}-02$ & $-6.8943336 E-03+j-8.9333279 E-02$ \\
\hline 18 & $1.7461762 E-02+j-5.3741777 E-02$ & $1.8959038 \mathrm{E}-02+\mathrm{j}-7.8211775 \mathrm{E}-02$ \\
\hline 19 & $1.9450958 \mathrm{E}-02+j-2.6771947 \mathrm{E}-02$ & $3.2868027 \mathrm{E}-02+\mathrm{j} \quad 4.8016421 \mathrm{E}-02$ \\
\hline 20 & $-6.2515354 \mathrm{E}-04+\mathrm{j} \quad 4.5420063 \mathrm{E}-04$ & $-3.7737216 \mathrm{E}-02+\mathrm{j}-3.3347617 \mathrm{E}-02$ \\
\hline 21 & $-2.3830421 E-02+j \quad 7.7429730 E-03$ & $3.6221968 \mathrm{E}-02+\mathrm{j} \quad 1.2016807 \mathrm{E}-02$ \\
\hline 22 & $-2.9786809 \mathrm{E}-02+\mathrm{j}-3.7033636 \mathrm{E}-16$ & $-3.0394661 E-02+j-3.1087231 E-03$ \\
\hline 23 & $-1.6758333 E-02+j-5.4451126 E-03$ & $2.2593241 \mathrm{E}-02+\mathrm{j}-7.4150625 \mathrm{E}-03$ \\
\hline 24 & $4.7466755 \mathrm{E}-04+\mathrm{j} \quad 3.4486616 \mathrm{E}-04$ & $-1.4831911 \mathrm{E}-02+\mathrm{j} \quad 8.5193392 \mathrm{E}-03$ \\
\hline 25 & $7.9736322 \mathrm{E}-03+\mathrm{j}$ & $8.1615065 \mathrm{E}-03+\mathrm{j}-1.1024794 \mathrm{E}-02$ \\
\hline 26 & $4.8990994 \mathrm{E}-03+\mathrm{j}$ & $-3.3539885 \mathrm{E}-03+\mathrm{j} \quad 7.7440547 \mathrm{E}-03$ \\
\hline 27 & $-2.1267981 E-16+j \quad 9.3326534 E-03$ & $3.5937439 \mathrm{E}-04+\mathrm{j}-7.0460161 \mathrm{E}-03$ \\
\hline 28 & $-7.3261783 \mathrm{E}-05+\mathrm{j} \quad 2.2547658 \mathrm{E}-04$ & $1.2415100 \mathrm{E}-03+\mathrm{j} \quad 3.1712087 \mathrm{E}-03$ \\
\hline 29 & $3.2850486 \mathrm{E}-03+\mathrm{j}-4.5214814 \mathrm{E}-03$ & $-1.1277025 \mathrm{E}-03+\mathrm{j}-2.1246454 \mathrm{E}-03$ \\
\hline 30 & $4.8331339 E-03+j-3.5114773 E-03$ & $4.6367180 \mathrm{E}-04+\mathrm{j} \quad 4.3466986 \mathrm{E}-04$ \\
\hline
\end{tabular}

and

$\boldsymbol{C}\left(\omega_{1}, \omega_{2}\right)=\left[\boldsymbol{C}_{0}^{\mathrm{T}}\left(\omega_{1}, \omega_{2}\right) \quad \boldsymbol{C}_{1}^{\mathrm{T}}\left(\omega_{1}, \omega_{2}\right) \quad \ldots \quad \boldsymbol{C}_{N-1}^{\mathrm{T}}\left(\omega_{1}, \omega_{2}\right)\right]^{\mathrm{T}}$,

where

$A_{i}=\left[\begin{array}{llll}a(i, 0) & a(i, 1) & \ldots & a(i, N-1)\end{array}\right]^{\mathrm{T}}, \quad 0 \leqslant i \leqslant N-1$,

and

$C_{i}\left(\omega_{1}, \omega_{2}\right)=\left[\begin{array}{llll}\mathrm{e}^{\mathrm{j} i \omega_{1}} & \mathrm{e}^{\mathrm{j} i \omega_{1}} \mathrm{e}^{\mathrm{j} \omega_{2}} \quad \ldots \quad \mathrm{e}^{\mathrm{j} i \omega_{1}} \mathrm{e}^{\mathrm{j}(N-1) \omega_{2}}\end{array}\right]^{\mathrm{T}}, \quad 0 \leqslant i \leqslant N-1$,

(35) can be rewritten as

$H\left(\omega_{1}, \omega_{2}\right)=\boldsymbol{A}^{\mathrm{T}} \boldsymbol{C}^{*}\left(\omega_{1}, \omega_{2}\right)=\boldsymbol{C}^{\mathrm{H}}\left(\omega_{1}, \omega_{2}\right) \boldsymbol{A}$,

which is used to approximate the arbitrary desired response $D\left(\omega_{1}, \omega_{2}\right)$. 




Fig. 2. Example 2: design of positive-sided differentiator. (a) Magnitude response.

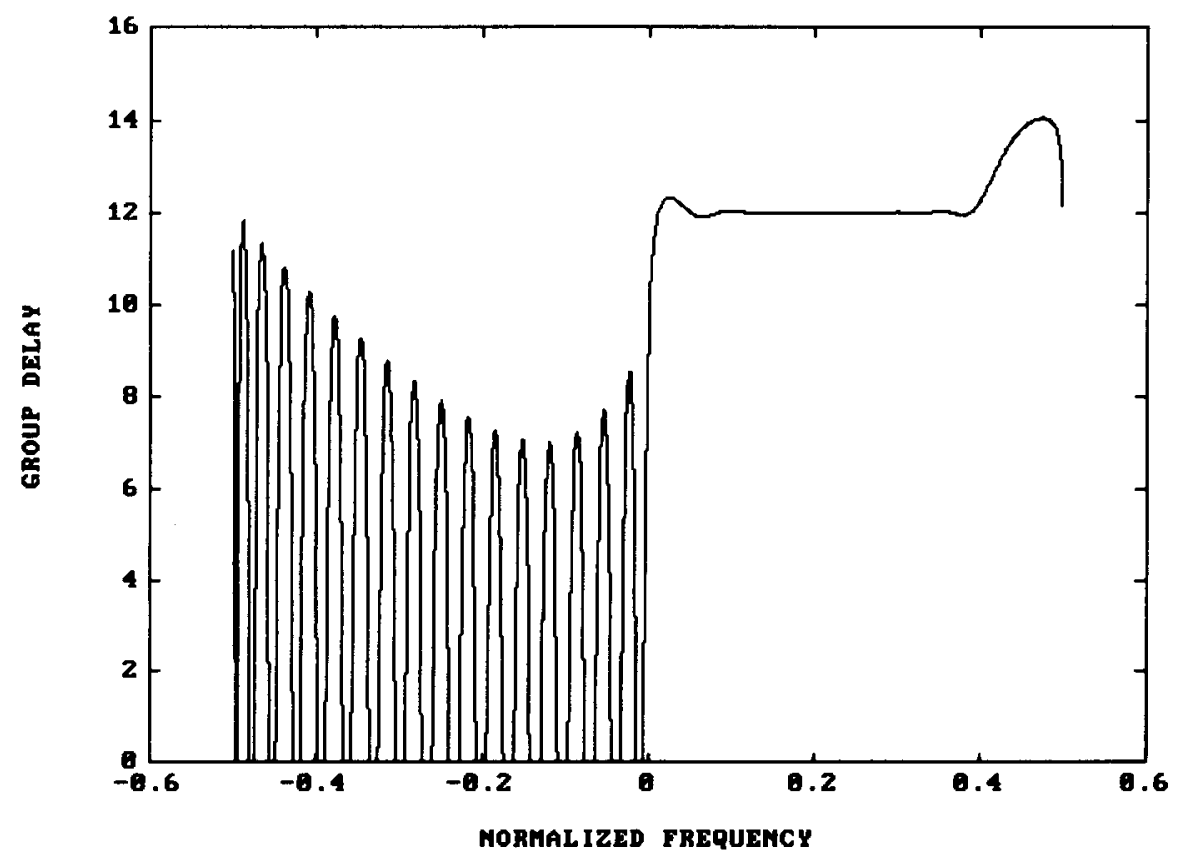

Fig. 2. Example 2. (b) Delay response. 


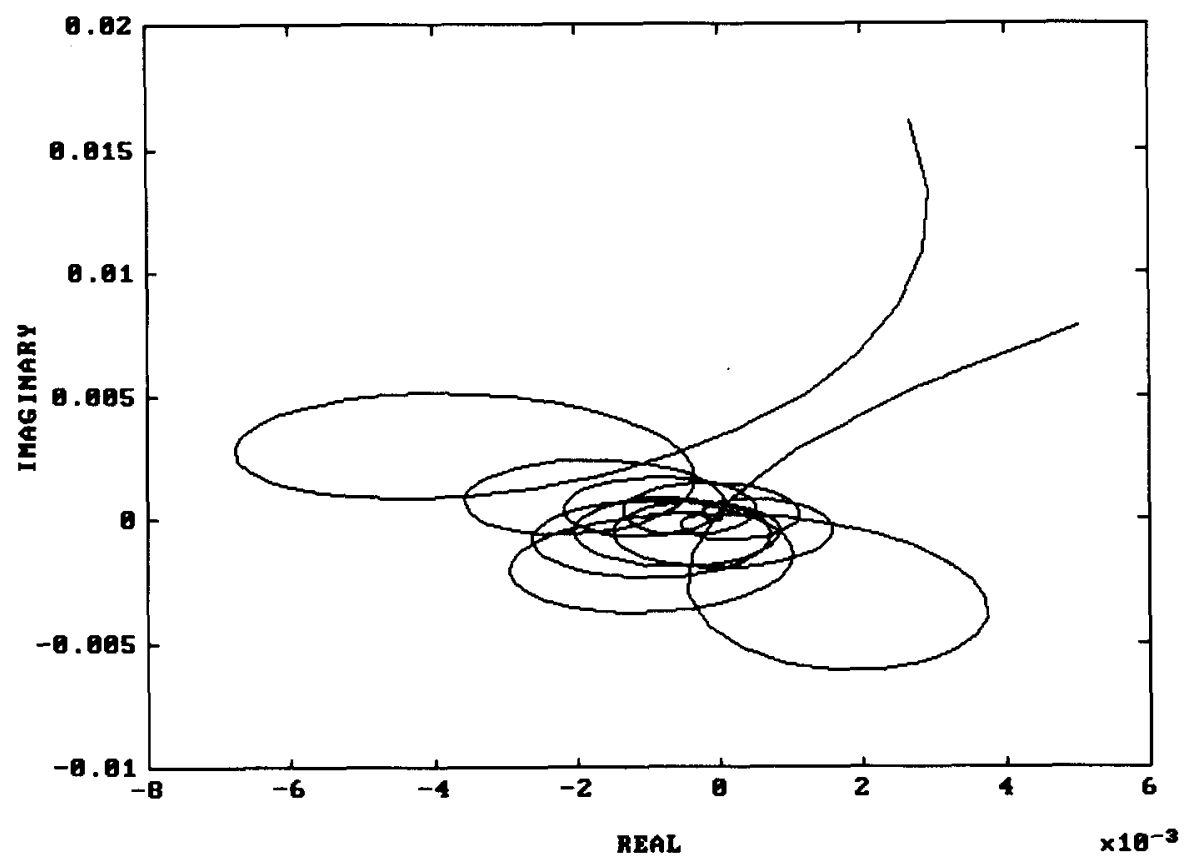

Fig. 2. Example 2. (c) Trace of passband complex error.

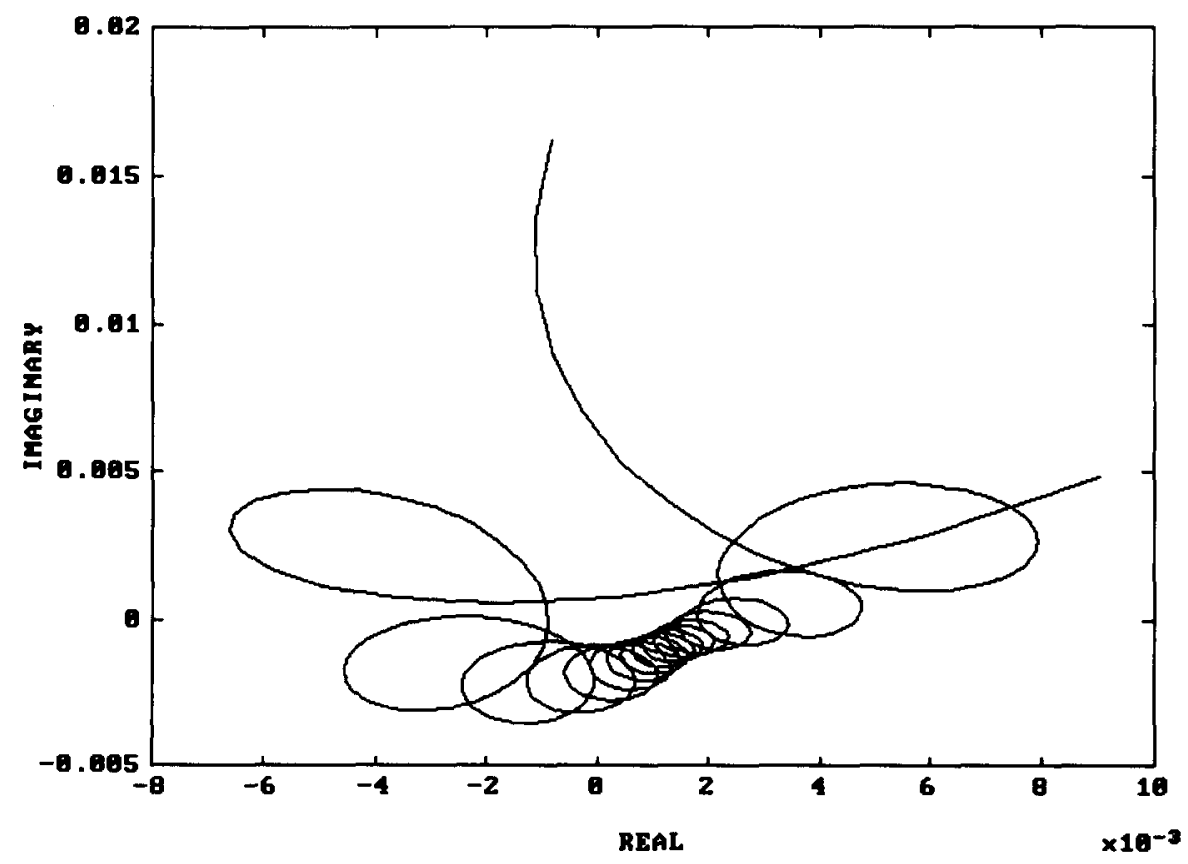

Fig. 2. Example 2. (d) Trace of stopband complex error. 


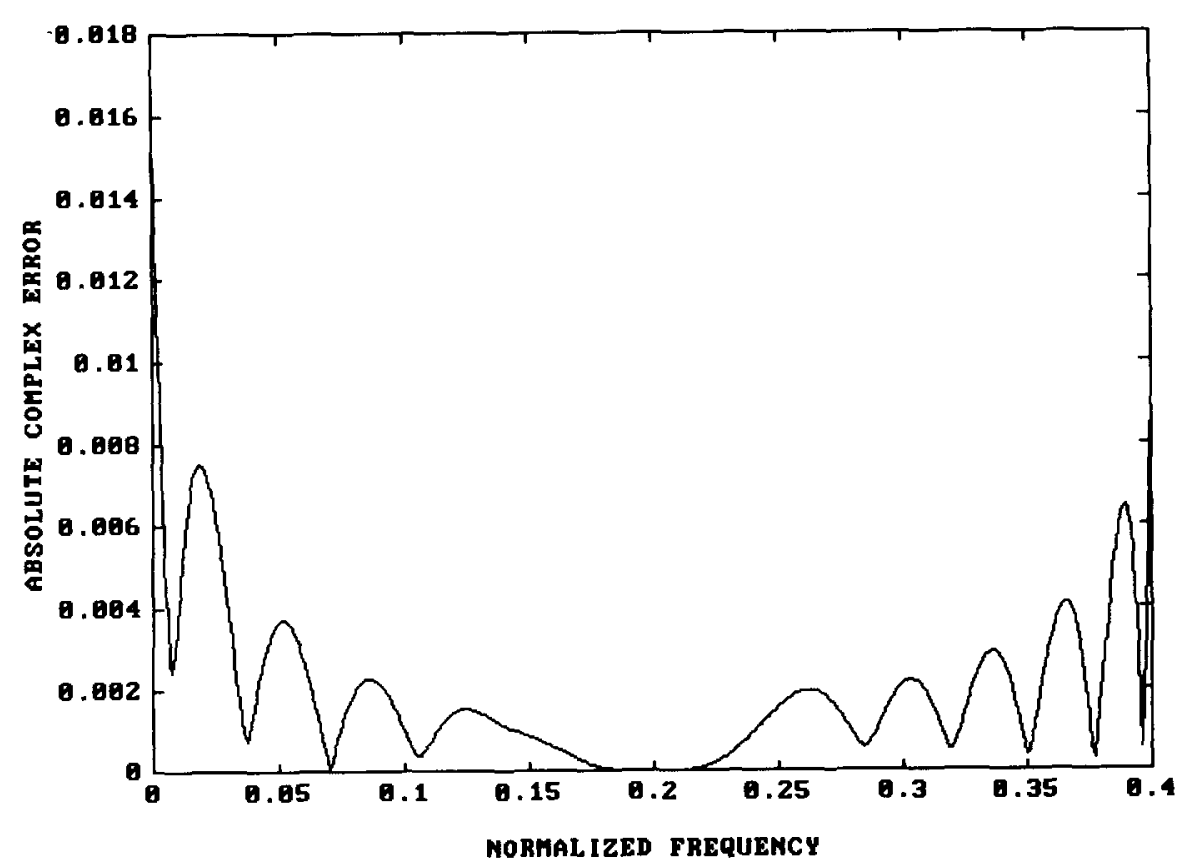

Fig. 2. Example 2. (e) Absolute passband complex error.

The integrated square complex error, which is similar to the one-dimensional case, can be formulated as

$e=s+P^{\mathrm{H}} \boldsymbol{A}+A^{\mathrm{H}} \boldsymbol{P}+A^{\mathrm{H}} \boldsymbol{Q} A$,

where

$s=\iint_{R_{\mathrm{p}}} \alpha D^{*}\left(\omega_{1}, \omega_{2}\right) D\left(\omega_{1}, \omega_{2}\right) \mathrm{d} \omega_{1} \mathrm{~d} \omega_{2}$,

$P=\iint_{R_{\mathrm{p}}}-\alpha D\left(\omega_{1}, \omega_{2}\right) C\left(\omega_{1}, \omega_{2}\right) \mathrm{d} \omega_{1} \mathrm{~d} \omega_{2}$

and

$\boldsymbol{Q}=\alpha \iint_{\boldsymbol{R}_{\mathrm{p}}} \boldsymbol{C}\left(\omega_{1}, \omega_{2}\right) \boldsymbol{C}^{\mathrm{H}}\left(\omega_{1}, \omega_{2}\right) \mathrm{d} \omega_{1} \mathrm{~d} \omega_{2}+\beta \iint_{\boldsymbol{R}_{\mathrm{s}}} \boldsymbol{C}\left(\omega_{1}, \omega_{2}\right) \boldsymbol{C}^{\mathrm{H}}\left(\omega_{1}, \omega_{2}\right) \mathrm{d} \omega_{1} \mathrm{~d} \omega_{2}$,

in which $\alpha, \beta$ are weighting constants and $R_{\mathrm{p}}, R_{\mathrm{s}}$ represent the passband and stopband regions, respectively.

Similarly, the desired two-dimensional filter passband performance is attained by imposing frequency response or derivative constraints $F$ at a discrete set of points $\left(\omega_{10}, \omega_{20}\right),\left(\omega_{11}, \omega_{21}\right), \ldots,\left(\omega_{1 J}, \omega_{2 J}\right)$ in the passband, i.e.

$F_{m j}\left[H\left(\omega_{1 j}, \omega_{2 j}\right)\right]=g_{m j}, \quad 0 \leqslant m \leqslant M$ and $0 \leqslant j \leqslant J$, 

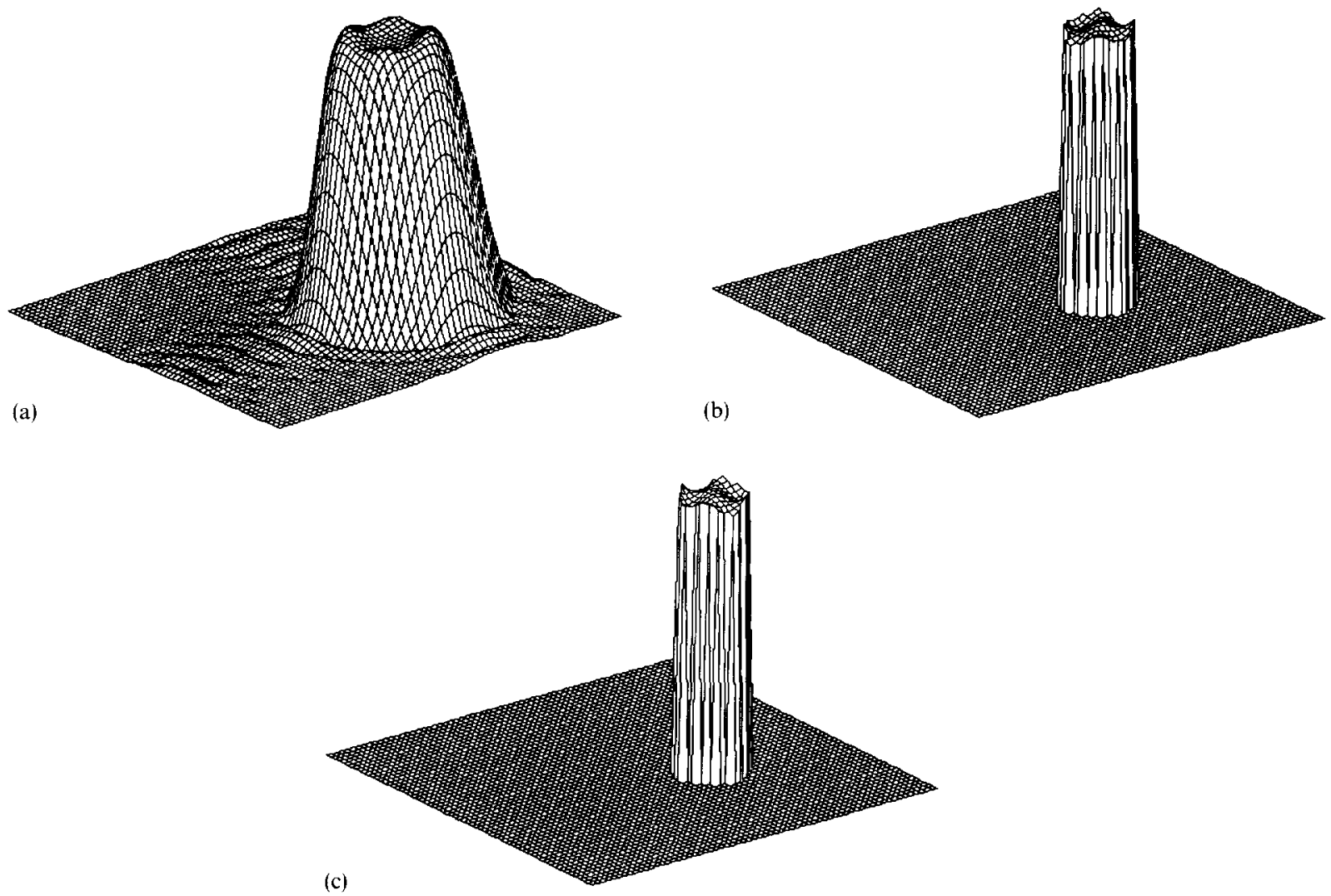

Fig. 3. Example 3: design of a circular-passband filter. (a) Magnitude response. (b) Delay response along the $\omega_{1}$-axis. (c) Delay response along the $\omega_{2}$-axis.

where $M$ denotes the order of constraints at a particular frequency $\left(\omega_{1 j}, \omega_{2 j}\right)$. Equation (45) can also be rewritten as

$B^{\mathrm{H}} A=G$

where

$\boldsymbol{B}=\left[F_{00}\left[\boldsymbol{C}\left(\omega_{1}, \omega_{2}\right)\right] \quad \ldots \quad F_{M_{0}}\left[\boldsymbol{C}\left(\omega_{1}, \omega_{2}\right)\right] \quad F_{01}\left[\boldsymbol{C}\left(\omega_{1}, \omega_{2}\right)\right] \quad \ldots \quad F_{M 1}\left[\boldsymbol{C}\left(\omega_{1}, \omega_{2}\right)\right] \quad \ldots\right.$

$\left.F_{0 J}\left[\boldsymbol{C}\left(\omega_{1}, \omega_{2}\right)\right] \quad \ldots \quad F_{M J}\left[\boldsymbol{C}\left(\omega_{1}, \omega_{2}\right)\right]\right]$

and the vector $G$ is the same as (18):

So the design problem for two-dimensional complex filters is the same as for the one-dimensional case, and the closed-form solutions are given in (25) and (26), but $I$ is an $N^{2} \times N^{2}$ identity matrix.

EXAMPLE 3. Design of a two-dimensional circular-passband filter.

The desired response is given by

$D\left(\omega_{1}, \omega_{2}\right)= \begin{cases}e^{-j \tau_{1} \omega_{1}} \mathrm{e}^{-\mathrm{j} \tau_{2} \omega_{2}}, & \sqrt{\left(\omega_{1}-\omega_{10}\right)^{2}+\left(\omega_{2}-\omega_{20}\right)^{2}} \leqslant \omega_{\mathrm{rp}}, \\ 0, & \sqrt{\left(\omega_{1}-\omega_{10}\right)^{2}+\left(\omega_{2}-\omega_{20}\right)^{2}} \geqslant \omega_{\mathrm{rs}},\end{cases}$ 
where $\tau_{1}$ and $\tau_{2}$ are the desired group-delay responses in the passband along the $\omega_{1}-$ and $\omega_{2}$-axis, respectively. Due to the nonseparable property for circular-region integration, the elements of $\boldsymbol{P}$ and $\boldsymbol{Q}$ can only be obtained by numerical methods. In this example, we use $N=11, \tau_{1}=\tau_{2}=4, \omega_{10}=0.2 \pi$, $\omega_{20}=0.3 \pi, \omega_{\mathrm{rp}}=0.3 \pi, \omega_{\mathrm{rs}}=0.5 \pi, \alpha=1, \beta=2$, and we hope to impose second-order derivative constraints on the desired frequency response at $\left(\omega_{10}, \omega_{20}\right)$, i.e.

$$
\begin{aligned}
& \boldsymbol{B}=\left[\mathrm{C}\left(\omega_{1}, \omega_{2}\right) \frac{\mathrm{d}}{\mathrm{d} \omega_{1}} \boldsymbol{C}\left(\omega_{1}, \omega_{2}\right) \quad \frac{\mathrm{d}}{\mathrm{d} \omega_{2}} \boldsymbol{C}\left(\omega_{1}, \omega_{2}\right) \quad \frac{\mathrm{d}^{2}}{\mathrm{~d} \omega_{1}^{2}} \boldsymbol{C}\left(\omega_{1}, \omega_{2}\right) \quad \frac{\mathrm{d}^{2}}{\mathrm{~d} \omega_{2}^{2}} \boldsymbol{C}\left(\omega_{1}, \omega_{2}\right)\right. \\
& \left.\frac{\mathrm{d}^{2}}{\mathrm{~d} \omega_{1} \mathrm{~d} \omega_{2}} \boldsymbol{C}\left(\omega_{1}, \omega_{2}\right)\right]\left.\right|_{\left(\omega_{1}, \omega_{2}\right)=\left(\omega_{10}, \omega_{20}\right)}
\end{aligned}
$$

and

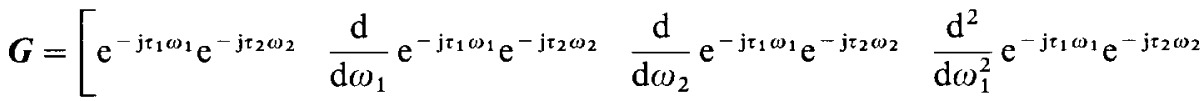

$$
\begin{aligned}
& \left.\frac{\mathrm{d}^{2}}{\mathrm{~d} \omega_{2}^{2}} \mathrm{e}^{-\mathrm{j} \tau_{1} \omega_{1}} \mathrm{e}^{-\mathrm{j} \tau_{2} \omega_{2}} \frac{\mathrm{d}^{2}}{\mathrm{~d} \omega_{1} \mathrm{~d} \omega_{2}} \mathrm{e}^{-\mathrm{j} \tau_{1} \omega_{1}} \mathrm{e}^{-\mathrm{j} \tau_{2} \omega_{2}}\right]\left.\right|_{\left(\omega_{1}, \omega_{2}\right)=\left(\omega_{10}, \omega_{20}\right)} .
\end{aligned}
$$

The resultant magnitude response is shown in Fig. 3(a), and the group-delay responses along the $\omega_{1}-$ and $\omega_{2}$-axis are shown in Figs. 3(b) and 3(c), respectively. Notice that the delay responses in the stopband in Figs. 3(b) and 3(c) have been set to zero.

\section{Conclusions}

A new technique has been proposed for the design of complex coefficient filters which approximate the arbitrary desired complex responses in this paper. The method is based on minimizing the integrated square complex error for the complex frequency response over the passband and stopband while imposing frequency response or derivative constraints. Also, the method can be extended to design two-dimensional FIR filters with arbitrary frequency response or derivative constraints. Several examples have been presented to demonstrate the effectiveness of the approach.

\section{References}

[1] C.-Y. Chi and S.-L. Chiou, "A new WLS Chebyshev approximation method for the design of FIR digital filters with arbitrary complex frequency response", Signal Processing, Vol. 29, No. 3, December 1992, pp. 335-347.

[2] S.M. Kay, Modern Spectral Estimation, Prentice Hall, Englewoods Cliffs, NJ, 1988.

[3] G.W. Medlin, "A design technique for high order digital differentiators", Proc. Internat. Conf. Acoust. Speech Signal Process., 1990, pp. $1285-1288$.

[4] G.W. Medlin and J.W. Adams, “A new technique for maximally linear differentiators”, Proc. Internat. Conf. Acoust. Speech Signal Process., 1989, pp. 825-828.

[5] G.W. Medlin and J.F. Kaiser, "Bandpass digital differentiator design using quadratic programming", Proc. Internat. Conf. Acoust. Speech Signal Process., 1991, pp. 1977-1980.

[6] G.W. Medlin, J.W. Adams and C.T. Leondes, "Lagrange multiplier approach to the design of FIR filters for multirate applications", IEEE Trans. Circuits and Systems, Vol. 35, October 1988, pp. 1210-1219.

[7] T.Q. Nguyen, "The design of arbitrary FIR digital filters using the eigenfilter method", IEEE Trans. Signal Process., March 1993, pp. $1128-1139$.

[8] S.C. Pei and J.J. Shyu, "Complex eigenfilter design of arbitrary complex coefficient FIR digital filters", IEEE Trans. Circuits and Systems - II, Vol. 40, January 1993, pp. 32-40.

Signal Processing 